\title{
Estimation Trajectory of the Low-Frequency Floating Car Considering the Traffic Control
}

\author{
Zhijian Wang, Min Li, Li Wang, and Xiaoming Liu \\ Beijing Key Lab of Urban Intelligent Traffic Control Technology, North China University of Technology, Beijing 100144, China \\ Correspondence should be addressed to Zhijian Wang; wzjian0722@163.com
}

Received 14 August 2013; Revised 30 October 2013; Accepted 18 November 2013

Academic Editor: Wuhong Wang

Copyright (C) 2013 Zhijian Wang et al. This is an open access article distributed under the Creative Commons Attribution License, which permits unrestricted use, distribution, and reproduction in any medium, provided the original work is properly cited.

\begin{abstract}
Floating car equipped with GPS to detect traffic flow has been widely used in ITS research and applications. The trajectory estimation is the most critical and complex part in the floating vehicle information processing system. However, the trajectory estimation would be more difficult when using the low-frequency data sampling because of the high communication cost and the numerous data. Specifically, the ordinary algorithm cannot determine the specific vehicle paths with two anchor points across multiple intersections. Considering the accuracy in map matching, this paper used a delay matching algorithm and studied the trajectory estimation algorithm focusing on the issue of existence of a small road network between two anchor points. A method considering the three multiobjective factors of signal control and driving distance and number of intersections was developed. Firstly, an optimal solution set was acquired according to multiobjective decision theory and Pareto optimal principles in game theory. Then, the optimal solution set was evaluated synthetically based on the fuzzy set theory. Finally, the candidate trajectory which is the core evaluation factor was identified as the best possible travel path. The algorithm was validated by using the real traffic data in Wangjing area of Beijing. The results showed that the algorithm can get a better trajectory estimation and provide more traffic information to traffic management department.
\end{abstract}

\section{Introduction}

With the rapid development of economic, travel efficiently tends to decline in recent years because of the congestion of traffic road network. Road travel time used in the dynamic traffic information service represents the key parameter of traffic congestion information. The quality and timeliness of road travel time determined the success of dynamic traffic guidance system. How to accurately acquire the travel time information of road network is very critical for the traffic guidance system. With the development of GPS (Global Positioning System) and GIS (Geographic Information System) technology, floating car system as a new traffic information acquisition method has the advantage as follows: short construction period, less investment, large range cover, high precision data, and better real time. Such systems have become a new acquisition method of dynamic traffic information instead of traffic fixed detector for high maintenance cost and big investment [1-3].

Although the floating car system as the dynamic information acquisition has a short history, it has become the research focus and is largely applied in the traffic control and traffic information service in foreign countries. In the famous ADVANCE project of American, the utilization of floating car system showed the importance in the traffic guidance system [4]. The German Aerospace Center built the floating car system based on the taxi, which can receive the mass GPS data to estimate the travel time for the traffic control center [5]. The Nagoya of Japanese has done the biggest experiment of floating car system in 2003-2007, which used the location information of 1,500 floating car to estimate and judge the real time traffic state of road network and publish the traffic information so as to distribute the traffic flow and ease the traffic congestion [6].

Some floating car system researches aimed at dealing with the urban traffic congestion were completed by the domestic traffic experts and scholars in recent years. The transportation research center of Beijing has built the road network traffic intelligent analysis system based on the 7,000 floating cars, which integrated the map matching and travel time estimation for road network state judgment and dynamic traffic information release through the real time position 


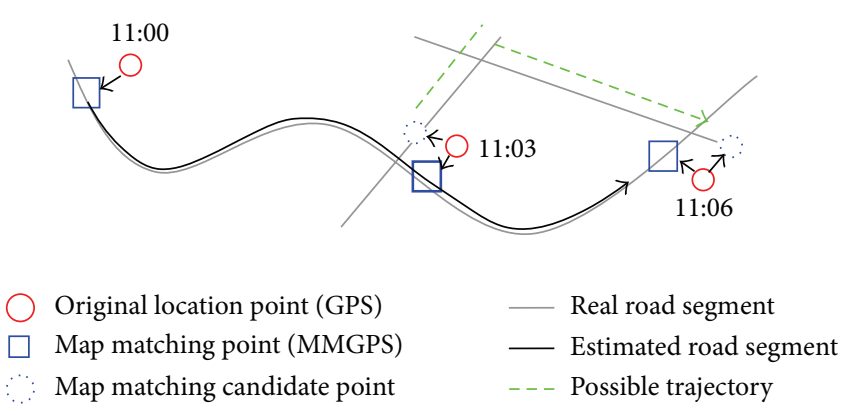

FIGURE 1: Map matching and trajectory estimation of Low-frequency GPS data.

platform [7]. Based on the 15,000 floating cars, Transport Commission of Guangzhou city built the intelligent navigation and location information service system for the 2010 Guangzhou Asian Games [8]. Similarity, Hangzhou city have installed the GPS for the 10,000 taxis to schedule the vehicle and road network state judgment, which also integrated the microwave detector data of road segment [9].

\section{Research and Analysis}

Although the floating car system was largely applied in traffic management, some difficulties still existed. The trajectory estimation of floating vehicles is an emphasis of the floating vehicle information processing system, in particular, in the case of using the low-frequency data sampling because of the high communication cost and significant data redundancy. This paper focuses on the trajectory estimation research, but firstly, there are two problems as follows.

2.1. The Accuracy of Map Matching before the Trajectory Estimation. In the floating vehicle information processing system, the result of accurate map matching is required before performing trajectory estimation and can largely affect the estimated results. Map matching algorithm has relation with the vehicle longitude and latitude, speed, vehicle travel angle, and the road topology composition. The floating car may run through some road segments in the GPS sampling period for the complicated topology between the adjacent GPS points. In addition, high-rise and viaduct occlusion cause the vehicle GPS data loss and frequent dynamic drift. As shown in Figure 1, if the anchor point was not accurately positioned after map matching, the trajectory estimation might come with two completely different travel paths.

2.2. The Difficulty of Estimating the Vehicle Trajectory. For the situation shown in Figures 2 and 3, if we connected simply with two anchor points, vehicle tracks will appear in the nonroad area. In Figure 2, we can replace the straight line p1p2 with the p1Ap2 as running track of the vehicle. However, the matched GPS point 1 and point 2 have a small network in Figure 3, and we need to apply the specific trajectory estimation method to determine the vehicle most possible route.

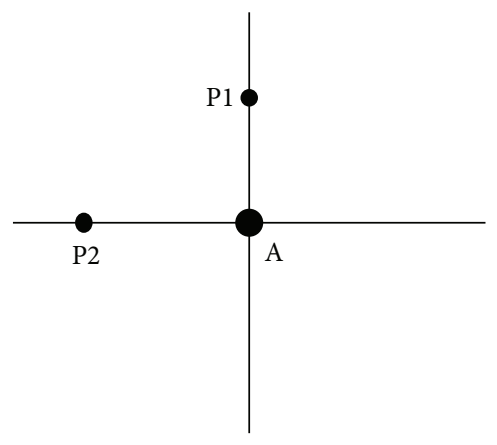

FIGURE 2: Two anchor points on the adjacent road.

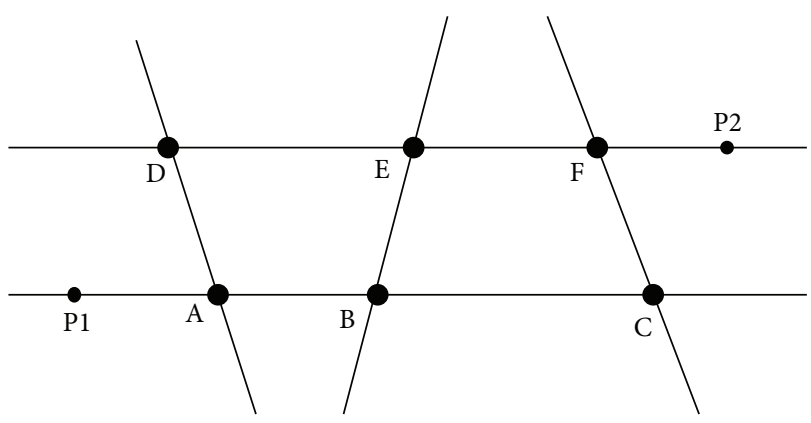

FIGURE 3: A small network between the matched GPS p1 and p2.

\section{Map Matching Algorithm}

For the first problem of research and analysis in the above, we know that the result of accurate map matching is essential before performing trajectory estimation. Map-matching algorithm based on the matching principle are mainly geometric matching algorithm, matching algorithm based on fuzzy logic, matching algorithm based on the cost function, and matching algorithm based on pattern recognition. For map-matching accuracy problems, White et al. [10] discussed some simple map matching algorithms that can be used to reconcile inaccurate location data with an inaccurate map/ network. Then he discussed point-to-point, point-to-curve, and curve-to-curve matching. But in some situations, directions do not change much as a result of small errors in the map matched location. Taylor et al. [11] proposed an improved map matching algorithm for GPS data in inaccurate conditions. The experiment of London to match the 15,000 sampling data can prove the higher accuracy algorithm. Based on analyzing the factors of affecting real time performance, robustness, and matching precision of the map matching algorithm and according to the continuity of the vehicle movement, the idea of partitioning the road net into some grids was introduced. Joshi [12] proposed a new approach to map matching for in-vehicle navigation systems. The paper presented a new metric, the rotational variation metric, which provided a new method for comparing vehicular and map paths for the purpose of map-matching. Quddus et al. [13] provided an improved map matching algorithm based on probability and statistics, the main features of which are the vehicle positioning error, the vehicle history trajectory, 


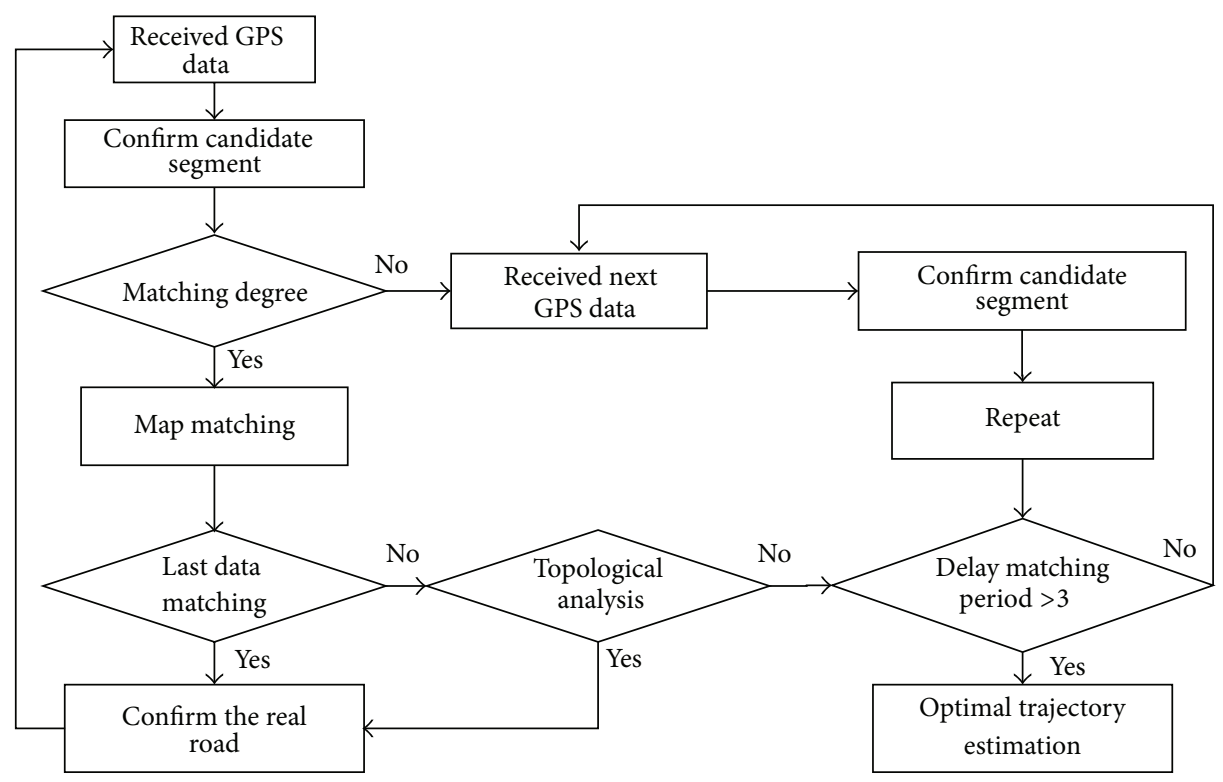

FIgURE 4: Chart of delay map matching algorithm.

and topological and vehicle speed and direction angle information all into account, solving the map matching when positioning data or the electronic map data was not accurate. In a word, now the map matching algorithm mainly aimed to solve the problem of the floating car in which region, which is influenced by the hall building occlusion and data loss. We need to determine the floating car driving in which road based on the topological relation and GPS data information so as to provide more accurate vehicle driving information.

The author $[14,15]$ has previously been employed the algorithm of delay map matching based on road topology structure which can be used when the vehicle cannot match the data at the first time. The actual testing data indicated that the new algorithm had a great instantaneity and accuracy in map matching, which effectively solved the problem of traffic data collection in complex intersection and area of viaduct bridge. The algorithm implementation and results are as in Figures 4, 5, 6, 7, and 8 .

\section{Trajectory Estimation}

4.1. Analysis of Study Methods. In the second problem of research and analysis in the above, the GPS positioning cycle is relatively long so the vehicle may run through the more road section. In Figure 3, the matched GPS point 1 and point 2 have a small network, in which the starting point and ending point have more than one path. In the situation we cannot confirm the floating car driver runs which route utilizing the related traffic information. So we need to apply the specific trajectory estimation method to determine the vehicle most possible route.

Takada [16] proposed the optimal floating car trajectory function, considering the location, estimation potential points, the driver preference, and travel characteristics which the traveler prefers to run the distance shorter route. Wang [8] proposed the trajectory estimation algorithm based on

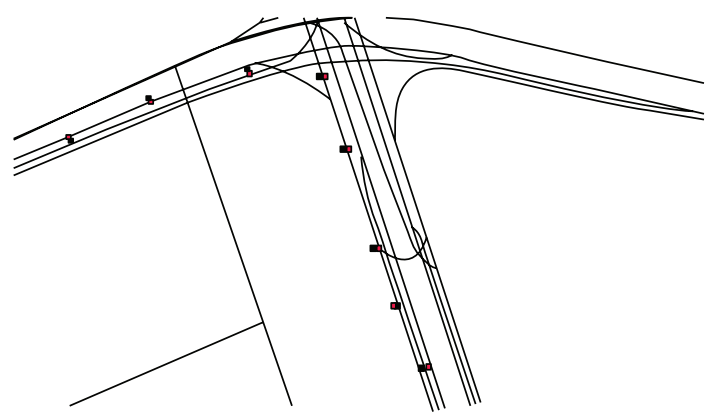

FIGURE 5: Point-map matching when around viaduct.

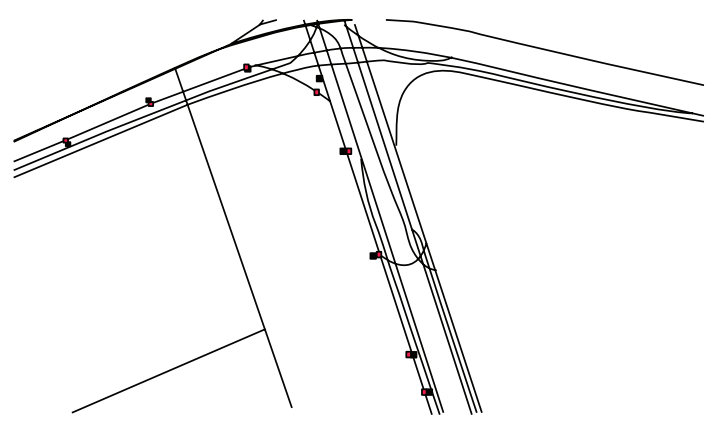

FIGURE 6: Delay map matching when around viaduct.

the mobile phone location data, considering the vehicle acceleration and deceleration to research the relationship of the travel time, which is according to the path searching to determine the vehicle trajectory. Chen [17] introduced Pareto multiobjective optimization and fuzzy theory to comprehensively judge the most likely path for each tracking problem. During this process, the driving distance and the number of passing crossroads were set as two optimal objectives. Then 


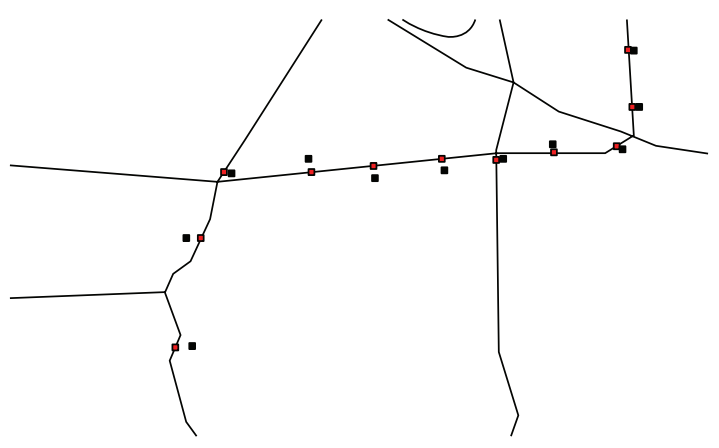

FIgURE 7: Point-map matching around intersection.

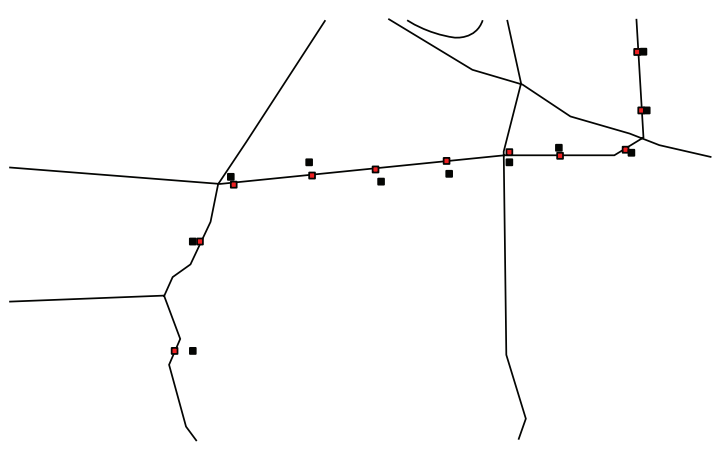

FIGURE 8: Delay map matching around intersection.

he distributed the average speed of each path proportionally to the road segments it covers, which integrated all the speed contributions a road segment collects for a final estimation of its traffic state. Kerner et al. [18] introduced a method for a reporting behavior at optimal costs of single vehicles (FCD: Floating Car Data) in road networks with the aim of a high quality of traffic state recognition which is presented. It is shown that based on minimum two FCD messages the substantial information of a typical traffic incident in a traffic center can be recognized. Byon et al. [19] introduced a method collecting and analysis traffic conditions of links by monitoring speed of probe vehicle(s) and then estimates travel time data both in static and dynamic modes. The static mode refers to the case of offline processing of GPS data from previously dispatched GPS-equipped vehicles to specified road links. The dynamic mode refers to the real time monitoring of speed on the links using a GPS-wireless Internet-equipped probe vehicle. In a word, the existing vehicle trajectory estimation algorithms usually do not consider the vehicles trajectory affected by signal control cycle, only considering the shortest path so the method is easy to cause an estimated error.

This paper focused on these issues that introduce a method of combining Pareto multiobjective optimization and fuzzy theory considering these three multiobjective factors of signal control, driving distance, and the number of intersections.

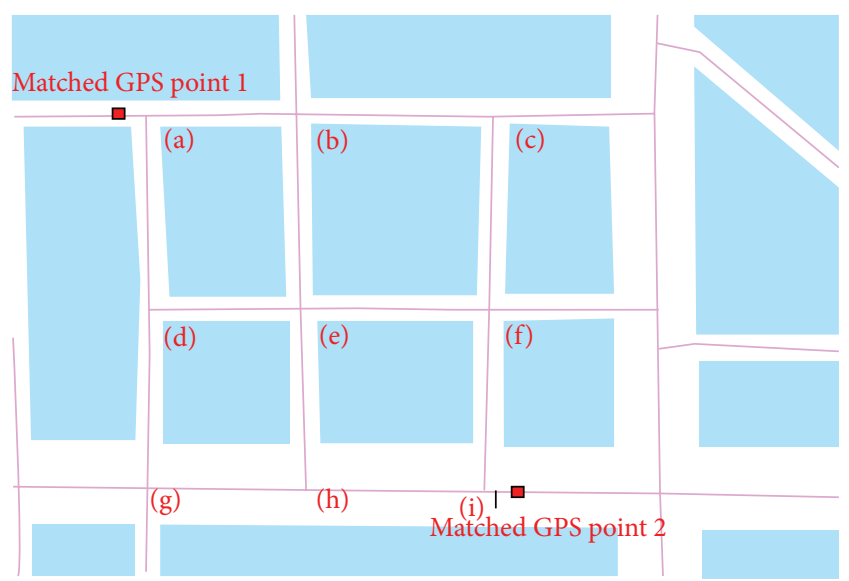

FIGURE 9: Actual road network of vehicle tracking.

\subsection{Multiobjective Decision Theory and Pareto Optimal Principles}

4.2.1. Multiobjective Decision Theory. When the decision object has a plurality of evaluation target, we can choose a satisfactory decision method from several feasible schemes (also known as the solution). According to the advance evaluation criterion we choose through the "priority" and "balance" to find a satisfactory solution from a set of nondominated solutions.

Multiobjective optimization problem was first developed by Italian economist Pareto L. put forward in 1896. In 1944 Von Neumann and Morgenstern [20] put forward conflicting multiobjective decision making with multiple decisionmakers from the perspective of game theory. Koopmans [21] in 1951 presented in multiobjective optimization problem from the production and distribution activities analysis and introduces the concept of Pareto optimization.

There are three possible results of possible options for a multiobjective decision problem: first, the solution in which all the objectives are the best is called complete optimal solution and this situation rarely occurs; second, the solution in which all the objectives are the worst is called the inferior solutions and can be immediately eliminated; third, the solution in which the objectives have good and bad goals is called noninferior solutions, also known as the Pareto optimal or efficient solution.

Multiobjective optimization mathematical model is assuming system has $y$ objectives $\prod_{1}(\omega), \prod_{2}(\omega), \ldots, \prod_{y}(\omega)$, the target vector $k=\left(k_{1}, k_{2}, \ldots, k_{y}\right)$ by the $y$ variables needs to be evaluated. If these objectives are required the maximum (or minimum) and required to satisfy the constraints set $R$, then the mathematical model can be expressed as $\max _{x \in R} F(x)$ or $\min _{x \in R} F(x)$, in which $F(\omega)=\left(\prod_{1}(\omega)\right.$, $\left.\prod_{2}(\omega), \ldots, \prod_{y}(\omega)\right)$.

Let us analyze the actual road network in Figure 9. The matched GPS point 1 and point 2 have more than 10 routes from which the driver can choose. What factor is the driver most likely to concern about when driving on the road network? According to the survey result, it is that arriving to the destination as soon as possible. Considering the influence 


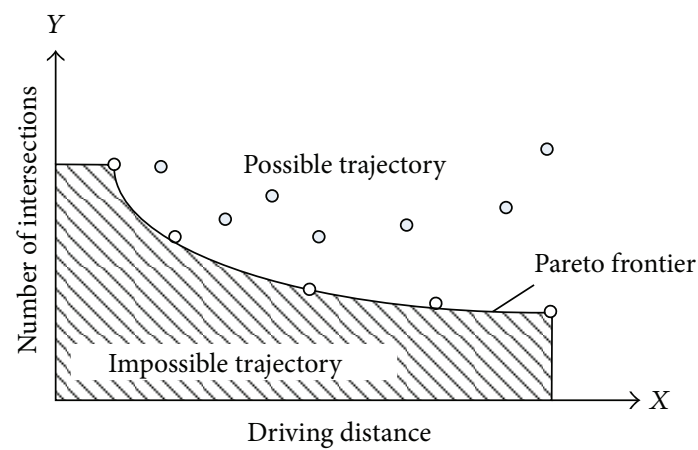

Figure 10: Vehicle possible trajectory in 2D plot of intersections number and driving distance.

of the floating car encountering the red-light delay time on the route choice, we ascertain the main factors of the vehicle trajectory judgement:

(1) travel the shortest distance as far as possible;

(2) as little as possible running through the intersections;

(3) as much as possible to reduce the delay time of the red light.

But when there are multiple objectives, because there is a conflict between the objectives that cannot be compared, it is difficult to find a solution so that all objective functions are simultaneously optimal. We know the drivers often go more some distance in order to avoid crossing the intersection. The detour distance which different drivers can accept is not the same and is influenced by the driver's preference and the actual traffic status. So we need to find the optimal using the principle of Pareto optimal solution.

4.2.2. Pareto Optimal Principles. Pareto optimality is an important concept in game theory. For multiobjective optimization problem, there is usually a solution set by which all these solutions in terms of the objective function are beyond comparison, called the Pareto optimal solution.

In our problem, in order to find floating car driving path of achieving the Pareto optimality, a three-dimensional coordinate space needs to be established as our solution space. The $x$-, $y$-, and $z$-axes, respectively, determine floating car traveling distance, the number of intersections, and the waiting time of intersections.

We can describe all possible paths solution to the coordinate space. The solution space can be decomposed into three two-dimensional solution planes which represent relationship of three objects by two and two shown in Figures 10,11 , and 12 . Shaded area is practically impossible to reach solution domains, namely, floating car driving distance, the number of intersections, and waiting time of intersections cannot simultaneously achieve such an ideal situation. All trajectories solution falling on the continuous curve can be considered as a set of optimal solutions. The curve is called Pareto Frontier of the current solution space, while the set of nondominated solutions is the Pareto optimal solution.

(1) From the large number of survey data, if we reached the destination more closely, the number of crossing

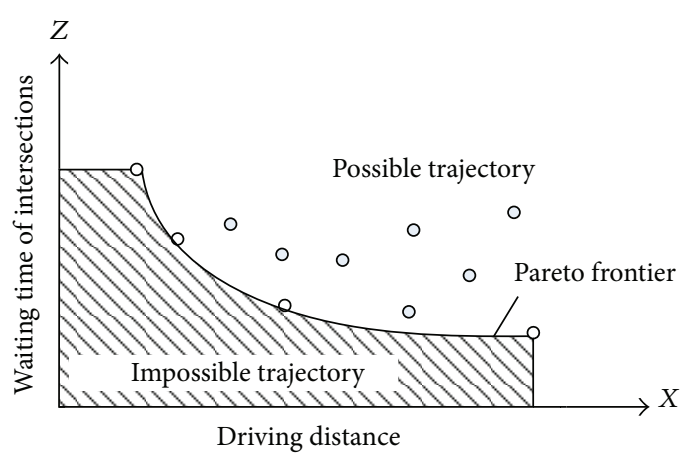

FIGURE 11: Vehicle possible trajectory in 2D plot of waiting time and driving distance.

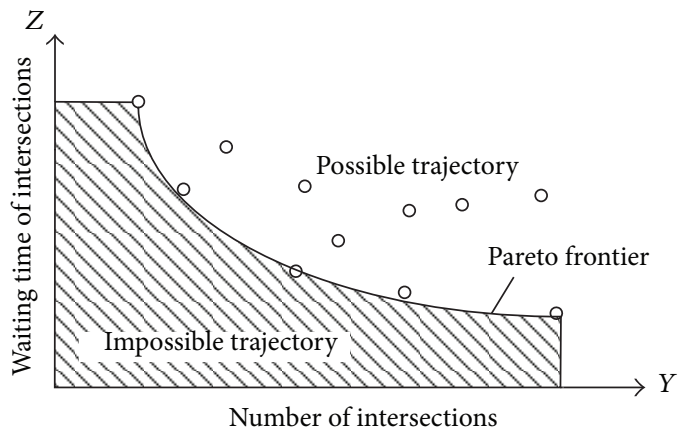

FIGURE 12: Vehicle possible trajectory in 2D plot of intersections number and waiting time.

the intersection may increase. On the contrary, the number would decrease. The number of intersections and driving distance of the floating car cannot simultaneously achieve the optimal in Figure 10.

(2) Also we analyze the survey data and find that if we reached the destination more closely, the intersection delay time may increase. On the contrary, the delay time may decrease. The relationship between delay time and driving distance is likely as the number of intersection and driving distance. This is a typical matter of choosing the shortest distance or the shortest time in Figure 11.

(3) As can be seen from the survey data, the number of intersection and waiting timing of crossing the intersection cannot simultaneously achieve optimal, but there is a Pareto Frontier in Figure 12.

4.3. Fuzzy Evaluation Analysis. Fuzzy comprehensive evaluation method is a comprehensive evaluation based on fuzzy mathematics method, and the method describes the fuzzy boundaries by fuzzy membership degree. The concept of fuzzy sets is proposed by the American Automatic Control Expert Chad (Zadeh) Professor [22] in 1965 to express the uncertainty of things.

In the paper, we utilize the fuzzy evaluation to analyze the driver's choice of travel trajectory, which can change the qualitative analysis into the quantitative analysis. Now we solve the problem of judging the vehicle trajectory when the 
adjacent GPS matched point has a small network. According to the travel distance, delay time of intersection, and the number of crossing intersections, we build the fuzzy set respectively.

4.3.1. Travel Distance Membership Function. According to the survey data, we set the $\mu_{d}$ as the travel distance function and build the discourse domain. The variables are the ratio of each path distance and the trajectory tracking problem of Euclidean distance between starting point and end point. We also define the parameter $\mu_{d 1} \mu_{d 2} \quad \mu_{d 3}$ as the membership function $\{$ (travel distance short), (travel distance medium), (travel distance long)\}. Membership functions are as follows:

$$
\begin{aligned}
& \mu_{d 1}= \begin{cases}1 & x \leq 1 \\
\frac{(1.3-x)}{0.3} & 1<x \leq 1.3 \\
0 & x>1.3\end{cases} \\
& \mu_{d 2}= \begin{cases}\frac{(x-1)}{0.3} & 1<x \leq 1.3 \\
\frac{(1.6-x)}{0.3} & 1.3<x \leq 1.6 \\
0 & x>1.6\end{cases} \\
& \mu_{d 5}= \begin{cases}0 & x \leq 1.3 \\
\frac{(x-1.3)}{0.3} & 1.3 \leq x \leq 1.6 \\
1 & x>1.6 .\end{cases}
\end{aligned}
$$

4.3.2. The Number of Intersections Membership Function. We set the $\mu_{n}$ as the number of intersections and build the discourse domain. We also define the parameter $\mu_{n 1} \quad \mu_{n 2} \quad \mu_{n 3}$ as the membership function $\{$ (intersection number less), (intersection medium), and (intersection number more) $\}$. According to the driving experience of floating car driver the number of intersections is not more than 6. So the membership functions are built as follows:

$$
\begin{aligned}
& \mu_{n 1}= \begin{cases}1 & y \leq 2 \\
2-\frac{y}{2} & 2<y \leq 4 \\
0 & y>4,\end{cases} \\
& \mu_{n 2}= \begin{cases}0 & y \leq 2 \\
\frac{y}{2}-1 & 2<y \leq 4 \\
3-\frac{y}{2} & 4<y \leq 6 \\
0 & y>6,\end{cases} \\
& \mu_{n 3}= \begin{cases}0 & y \leq 4 \\
2-\frac{y}{2} & 4<y \leq 6 \\
1 & y>6 .\end{cases}
\end{aligned}
$$

4.3.3. Delay Time of Intersection Membership Function. According to the survey data, we set the $\mu_{t}$ as the delay time function and build the discourse domain. We also define the parameter $\mu_{t 1} \quad \mu_{t 2} \quad \mu_{t 3}$ as the membership function \{(delay time short), (delay time secondary), and (delay time long)\}. According to the driving experience of floating car driver, delay time of intersection is not more than 3 minutes. So the membership functions are built as follows:

$$
\begin{aligned}
& \mu_{t 1}= \begin{cases}1 & z \leq 60 \\
2-\frac{z}{60} & 60<z \leq 120 \\
0 & z>120,\end{cases} \\
& \mu_{t 2}= \begin{cases}0 & z \leq 60 \\
\frac{z}{60}-1 & 60<z \leq 120 \\
3-\frac{z}{60} & 120<z \leq 180 \\
0 & z>180,\end{cases} \\
& \mu_{t 3}= \begin{cases}0 & z \leq 120 \\
2-\frac{z}{60} & 120<z \leq 180 \\
1 & z>180 .\end{cases}
\end{aligned}
$$

4.3.4. The Fuzzy Matrix Computation. After establishment of fuzzy membership function, we need to compute the fuzzy matrix to confirm the most possible vehicle trajectory. According to the fuzzy vector set as follows: $\mu_{d}=\left\{\mu_{d 1}\right.$ $\left.\mu_{d 2} \quad \mu_{d 3}\right\}, \mu_{n}=\left\{\begin{array}{lll}\mu_{n 1} & \mu_{n 2} & \mu_{n 3}\end{array}\right\}, \mu_{t}=\left\{\begin{array}{lll}\mu_{t 1} & \mu_{t 2} & \mu_{t 3}\end{array}\right\}$, we combine the fuzzy vector together and get the fuzzy matrix $R=$ $\left\{\mu_{d}^{T}, \mu_{n}^{T}, \mu_{t}^{T}\right\}^{T}$ considering the driver's different preferences on the road distance, delay time, and the number of intersections. So we build the weight vector $p=\left\{p_{d}, p_{n}, p_{t}\right\}$, where the relationship equation is $p_{d}+p_{n}+p_{t}=1$. Then we need to build the fuzzy vector $Q=P R$, in which the possibility of the vehicle trajectory represented by the each element is large, medium, or small. Furthermore, In order to determine the actual path, we introduce the matching degree of vectors $\lambda=\left\{\begin{array}{lll}\lambda_{1} & \lambda_{2} & \lambda_{3}\end{array}\right\} \quad\left(\lambda_{1}+\lambda_{2}+\lambda_{3}=1\right)$, by $\alpha=Q \lambda^{T}$, calculated for each path of the evaluation factors $\alpha$ (the possibility of a true path).

\section{Experimental Analysis}

To verify this algorithm of vehicle trajectory estimation, we choose an actual road based on the Beijing traffic control system to acquire the delay time of intersection in real time. We know that the vehicle speed, road distance, and delay time of intersection are affected by the traffic control. So, it is necessary to consider the impact of intersection signal control when we estimate the vehicle trajectory. Although the intersection signal control information is often difficult to obtain, this paper can rely Beijing traffic control information platform in real time to obtain the appropriate traffic control 


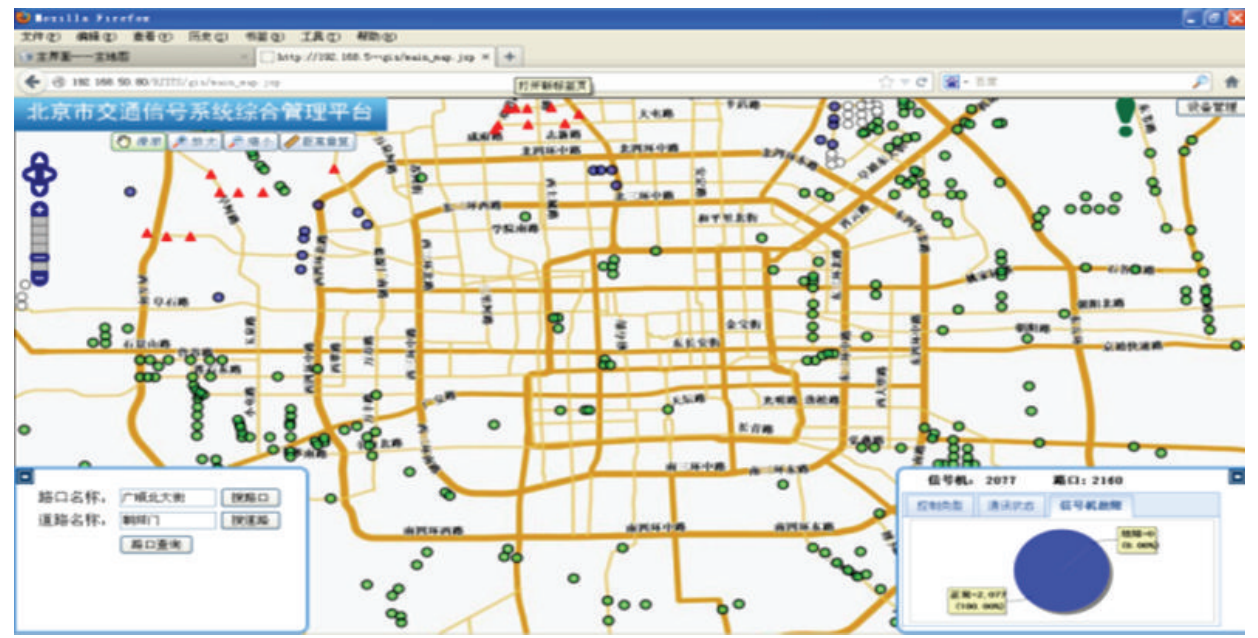

FIGURE 13: Beijing traffic control information platform.

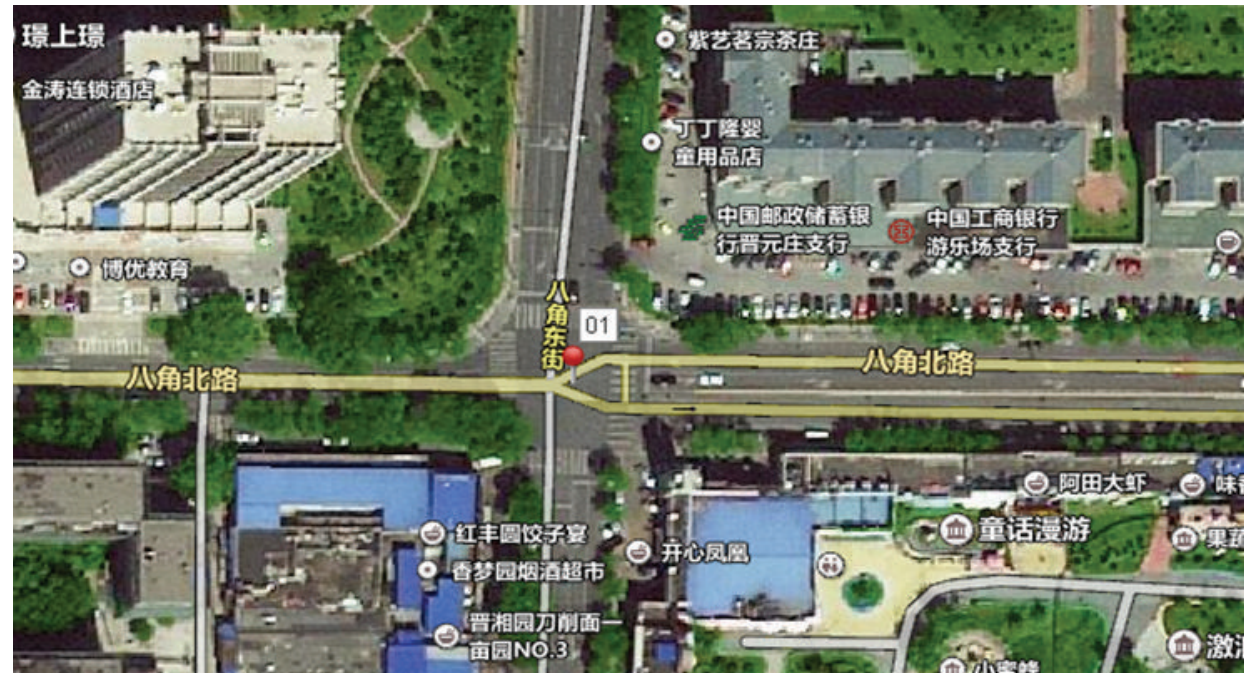

FIGURE 14: An intersection in Shijingshan district.

information. Figures $13,14,15$, and 16 are real time traffic timing of an intersection in Shijingshan district.

Here we determine the optimal path for an example of the urban road network in Wangjing area, Beijing city, shown in Figure 17, using the Pareto optimization and fuzzy comprehensive judgment method. The points P1 and P2 in the Figure are the starting point and end point of the undetermined path and there is a small road network which is connected by the intersections of A I between P1 and P2. Various sections of the road network length are marked in Arabic numerals (in meters) and some intersections with traffic lights are the light controlled intersections in the figure. So we can describe all possible paths from the point P1 to P2 to a three-dimensional coordinate space be composed of traveling distance, the number of intersections, and the waiting time.

The length data of all the road section from Figure 17 is as shown in Table 1.
The light controlled intersections with traffic lights in the Figure 17 cause vehicle delay time when the vehicle passes through the intersection and have a great impact on selecting the running track for drivers. So obtaining signal control information of each intersection is very necessary. We just need to get the timing cycle data of each intersection to determine the influence of the control signal in order to improve the computational efficiency (see Table 2).

But because of different ways of the vehicle through the intersection the impact of signals control is also different. For example, the control of traffic lights is different for vehicles turning left, going straight, and turning right, and the delay caused by the intersection is also different. So when we consider the impact of lights on the path selection, we need to consider the travel direction of the path in the intersection. The average delay caused by the different traveling direction can be determined by the intersection timing cycle. If we let $C$ as the cycle, we can set straight delay $t_{s}=C / 4$, left delay 
TABLE 1: The sections length for analytical experiment.

\begin{tabular}{|c|c|c|c|c|c|c|c|c|c|}
\hline \multirow{2}{*}{$D L(\mathrm{~m})$} & \multicolumn{9}{|c|}{$O L(\mathrm{~m})$} \\
\hline & A & B & C & D & $\mathrm{E}$ & $\mathrm{F}$ & G & $\mathrm{H}$ & I \\
\hline $\mathrm{A}$ & 0 & 400 & 640 & 350 & 1 & 1 & 1 & 1 & 1 \\
\hline B & 400 & 0 & I & I & 320 & I & 1 & I & 1 \\
\hline C & 640 & I & 0 & 400 & I & 410 & 1 & I & I \\
\hline $\mathrm{D}$ & 350 & 1 & 400 & 0 & 390 & 740 & I & I & I \\
\hline E & 1 & 320 & 1 & 390 & 0 & 1 & 1 & I & 410 \\
\hline $\mathrm{F}$ & I & 1 & 410 & 740 & 1 & 0 & 160 & 1 & I \\
\hline G & 1 & I & 1 & I & 1 & 160 & 0 & 480 & I \\
\hline $\mathrm{H}$ & 1 & 1 & 1 & 1 & 1 & I & 480 & 0 & 410 \\
\hline I & I & 1 & I & I & 410 & 1 & 1 & 410 & 0 \\
\hline
\end{tabular}

O: origin node, $D$ : destination node, $L$ : the sections length, /: nonexistent sections.

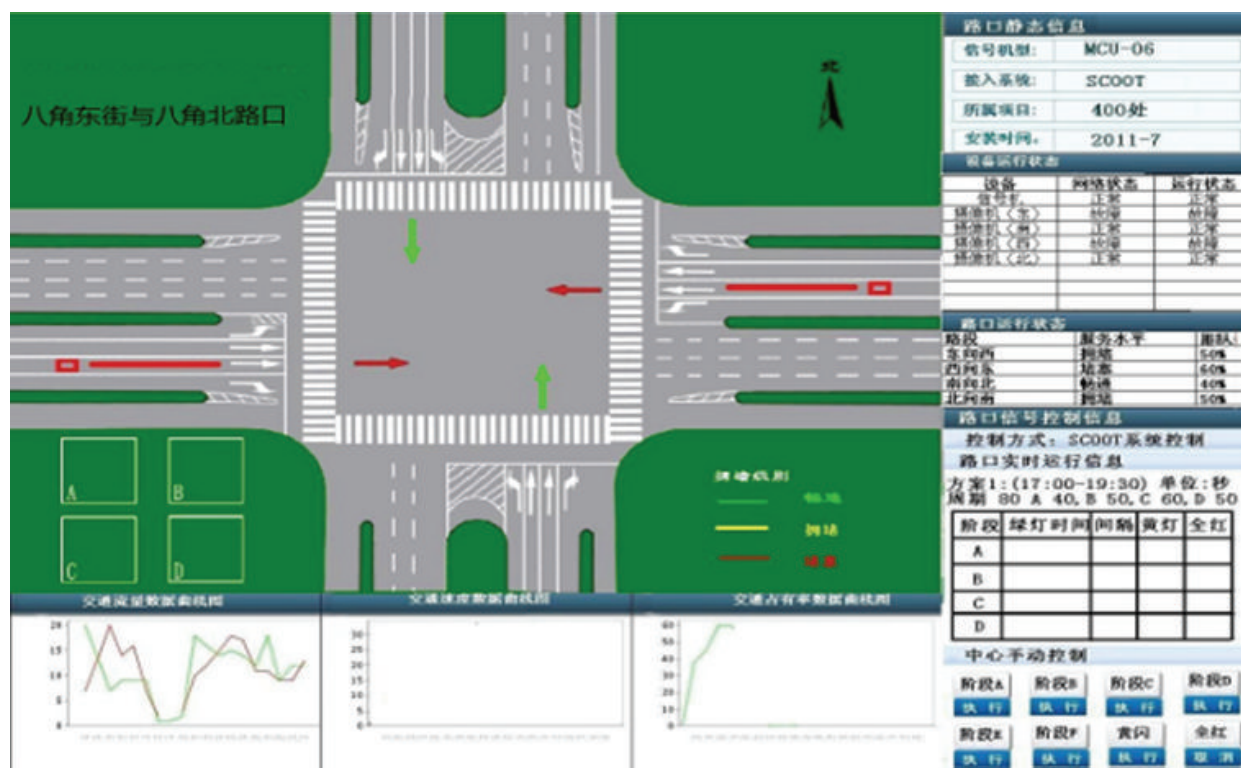

FIGURE 15: The traffic signal time program of the intersection.

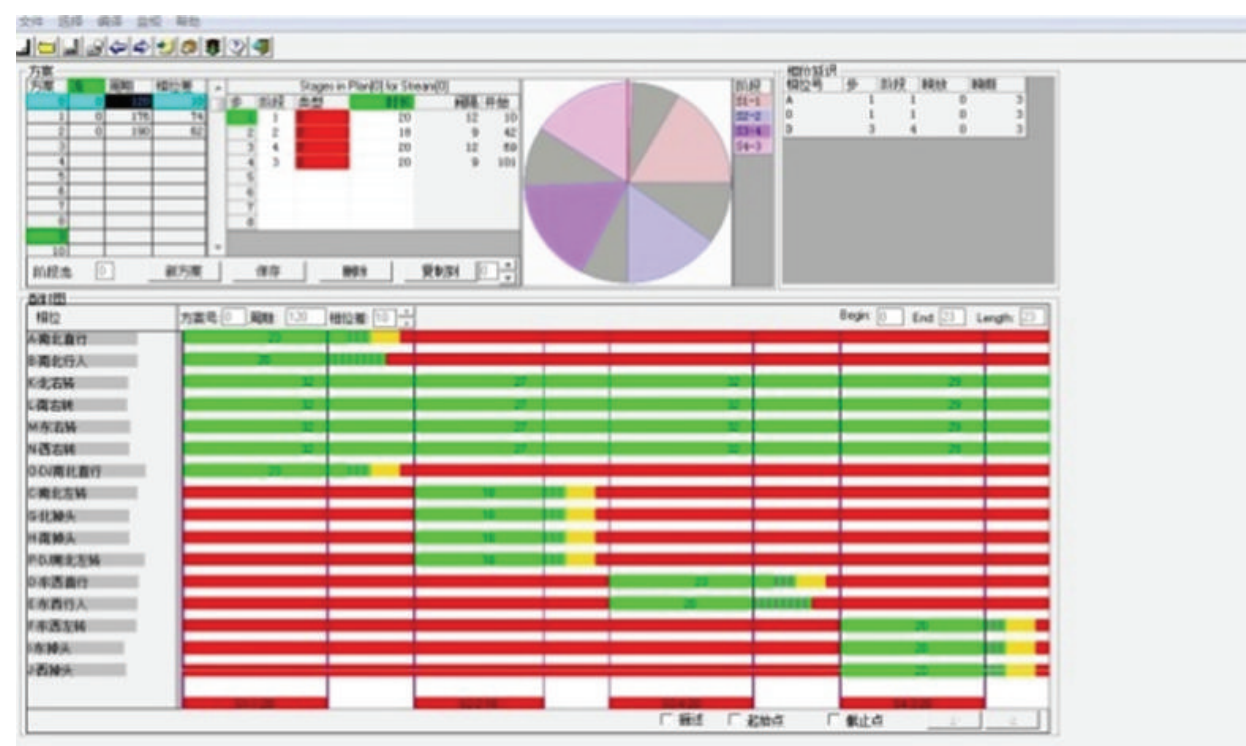

FIgURE 16: The timing plan of the intersection. 


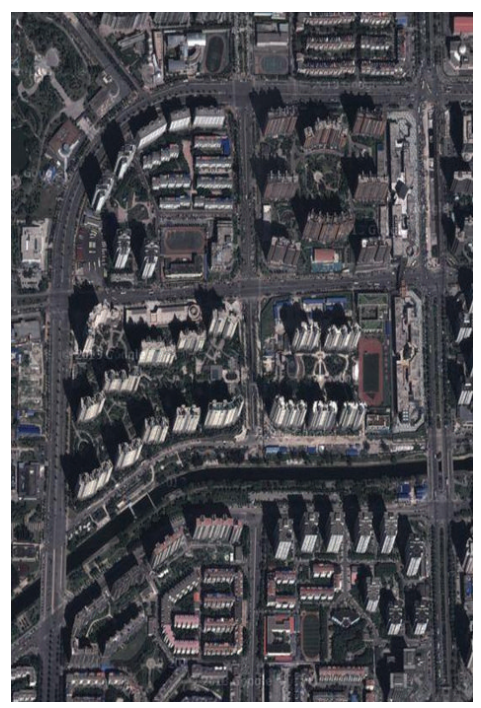

(a)

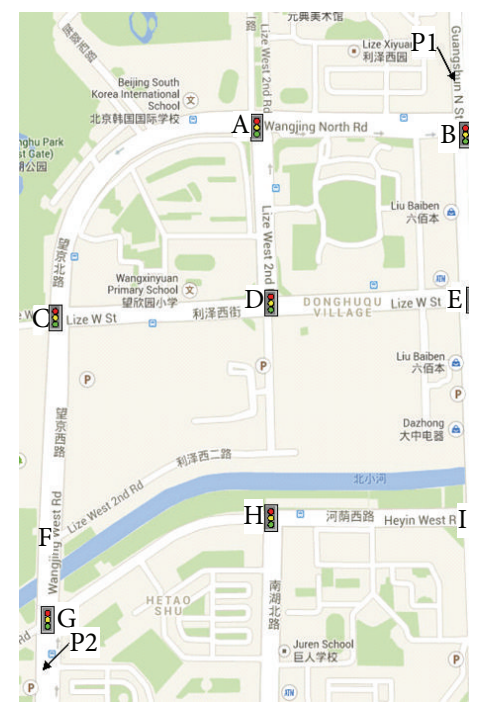

(b)

FIGURE 17: Wangjing area road network for analytical experiment.

TABLE 2: Signal timing cycle of each intersection.

\begin{tabular}{ccccccccc}
\hline & \multicolumn{8}{c}{ The light controlled intersections } \\
& A & B & C & D & E & G & H & I \\
\hline cycle(s) & 130 & 160 & 150 & 120 & 160 & 130 & 110 & 160 \\
\hline
\end{tabular}

$t_{l}=3 C / 8$, and right delay $t_{r}=0$ (without considering the influence of signal control, only considering the impact of the intersections number).

The possible paths obtained by using the path search method are drawn in three-dimensional space composed of floating car traveling distance, the number of intersections, and the waiting time of intersections (Table 3 ).

Then we can obtain Pareto optimal solution using multiobjective decision theory and Pareto optimal principles, and this group of the optimal solution set forms the Pareto Frontier of the current optimization problem. The indeterminate trajectories in Pareto frontier are the numbers 1, 2, 4, and 6 that is B-A-C-F-G, B-A-D-F-G, B-E-D-F-G, and B-E-I-H-G. Then we can choose the best trajectory from the four paths on Fuzzy Comprehensive (Table 4).

Here we choose the similar weight of evaluating indicator for travel distance, intersection number, and delay time, so the weight vector $P=\left[\begin{array}{lll}0.3 & 0.3 & 0.4\end{array}\right]$. Because the match degree of a path (the possibility of it as a true path) increases along with the decrease of traveling distance, the number of intersections, and the waiting time, we can construct the matching degree vector $\lambda=\left[\begin{array}{lll}0.6 & 0.3 & 0.1\end{array}\right]$.

According to the judging indicators in Table 5, we can find that the first candidate trajectory's $\alpha_{1}$ is the largest so we judge that the no. 1 path (B-A-C-F-G) is the most possible trajectory. We have done lots of the real experiment which the result showed that the driving car equipped GPS having the probability of 92 percentage selected the real driving

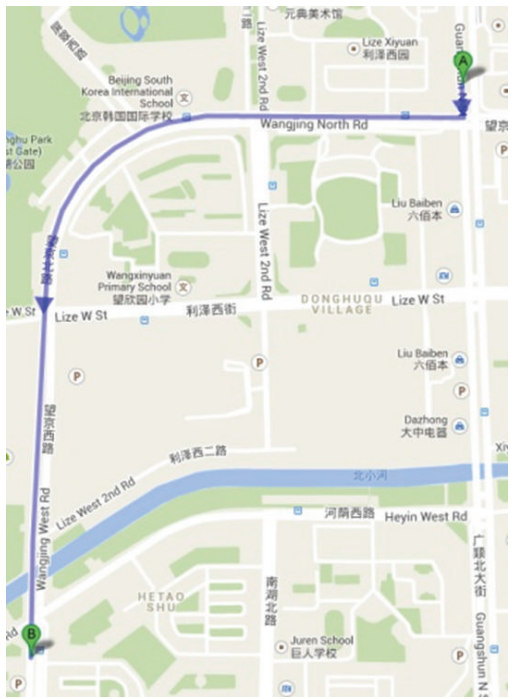

FIgURE 18: The most possible trajectory considering the traffic control.

trajectory comparison to the estimation trajectory by the algorithm introduced in the paper (Figure 18).

\section{Conclusion}

The efficient use of the floating cars data with the tracking of low-frequency sampling floating cars and traffic signal control is becoming a new hot topic. The overlong period of GPS sampling and overly complex road topology, which is the two features of current urban floating cars construction system, made it possible to use the floating car technology in transportation area. According to the map matching algorithm result, this paper developed a new algorithm based on 
TABLE 3: The possible trajectory data.

\begin{tabular}{|c|c|c|c|c|}
\hline & Possible trajectory & Distance (meters) & Intersection numbers & Delay time (s) \\
\hline 1 & B-A-C-F-G & 1610 & 5 & $0+C_{A} / 4+C_{C} / 4+0+C_{G} / 4=102.5$ \\
\hline 2 & B-A-D-F-G & 1650 & 5 & $0+3 C_{A} / 8+C_{D} / 4+0+C_{G} / 4=111.25$ \\
\hline 3 & B-A-D-C-F-G & 1720 & 6 & $0+3 C_{A} / 8+0+3 C_{C} / 8+0+C_{G} / 4=137.5$ \\
\hline 4 & B-E-D-F-G & 1610 & 5 & $C_{B} / 4+0+3 C_{D} / 8+0+C_{G} / 4=117.5$ \\
\hline 5 & B-E-D-C-F-G & 1680 & 6 & $C_{B} / 4+0+C_{D} / 4+3 C_{C} / 8+0+C_{G} / 4=158.75$ \\
\hline 6 & B-E-I-H-G & 1620 & 5 & $C_{B} / 4+C_{E} / 4+0+C_{H} / 4+3 C_{G} / 8=156.25$ \\
\hline
\end{tabular}

TABLE 4: Fuzzy matrix of membership for candidate paths.

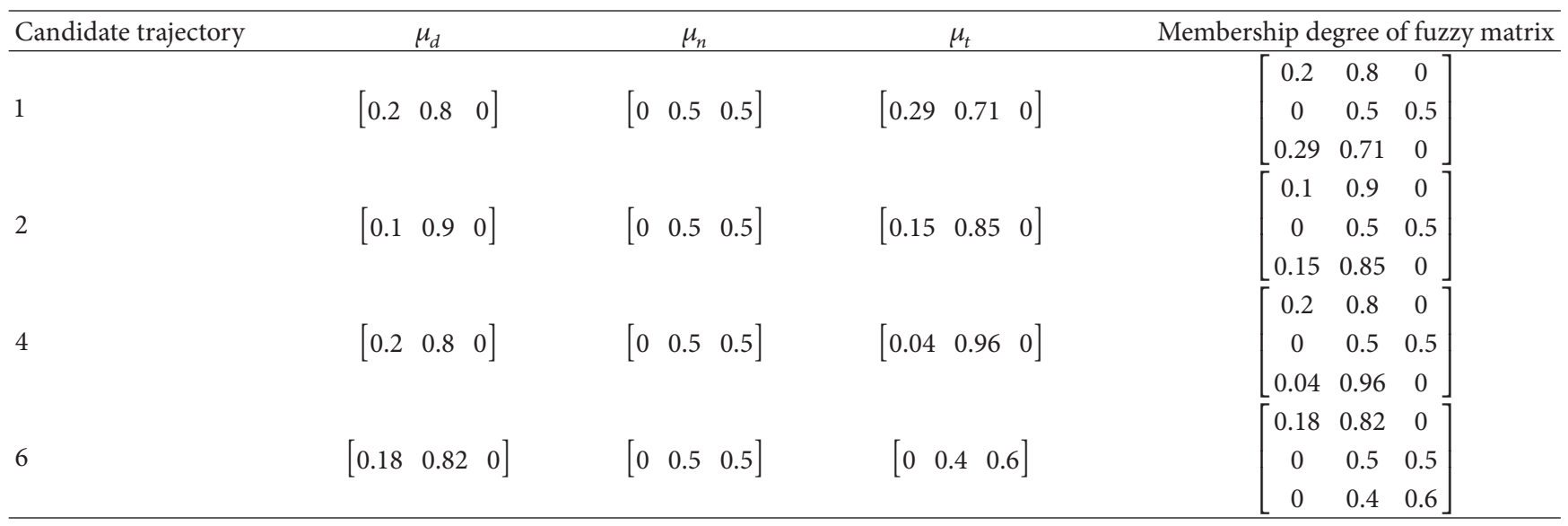

TABLE 5: Judging indicators of candidate paths.

\begin{tabular}{lcc}
\hline $\begin{array}{l}\text { Candidate } \\
\text { trajectory }\end{array}$ & The fuzzy vector $Q=$ & $\begin{array}{c}\text { The evaluation factor } \\
\alpha=Q \lambda^{T}\end{array}$ \\
\hline 1 & {$\left[\begin{array}{lll}0.18 & 0.67 & 0.15\end{array}\right]$} & 0.324 \\
2 & {$\left[\begin{array}{lll}0.09 & 0.76 & 0.15\end{array}\right]$} & 0.297 \\
4 & {$\left[\begin{array}{lll}0.08 & 0.77 & 0.15\end{array}\right]$} & 0.294 \\
6 & {$\left[\begin{array}{lll}0.05 & 0.56 & 0.39\end{array}\right]$} & 0.237 \\
\hline
\end{tabular}

multiobjective optimization and fuzzy theory by analyzing the characteristics of GPS data, which also integrated the trajectory estimating, driving distance, intersection time delay, and the crossroads number between the GPS matching points. The validation of the algorithm included the floating cars' trajectory estimating and the data of the signal control system. The result of the experiment achieved the author's prospective target and solved the questions mentioned before effectively. In present study, the author only conducts the experiment, estimating the floating cars' trajectory localized in urban. Therefore there are certainly some difficulties if tracking and estimating the vehicle moving no limitation in urban. In addition, because of the limited time and the length of the paper the path search algorithm and the Pareto frontier search algorithm of three-dimensional space are not efficient enough and are needed to be researched further.

However, the algorithm in the paper is reasonable and practical. The achievement of this paper is the further study of the application of floating cars' information which promote the traffic collection information technology effectively and will lay a solid foundation to the distribution and forecasting of floating cars' travel time.

\section{Conflict of Interests}

The authors declare that they do not have any commercial or associative interests that represent a conflict of interests in connection with the work submitted.

\section{Acknowledgments}

This study was supported by the National High Technology Research and Development Program of China (863 Program) (no. 2012AA112401), Beijing Talents Qualification Project (no. 2012D005002000006), and the Importation and Development of High-Caliber Talents Project of Beijing Municipal Institution (CIT\&TCD201304002).

\section{References}

[1] W. Wang, W. Zhang, H. Guo, H. Bubb, and K. Ikeuchi, "A safetybased approaching behavioural model with various driving characteristics," Transportation Research Part C, vol. 19, no. 6, pp. 1202-1214, 2011.

[2] W. Wang, X. Jiang, S. Xia, and Q. Cao, "Incident tree model and incident tree analysis method for quantified risk assessment: an in-depth accident study in traffic operation," Safety Science, vol. 48, no. 10, pp. 1248-1262, 2010.

[3] W. Wang and F. Hou, "Simulation-based dependability evaluation of complex repairable system using variable time increment method," in Proceedings of the 5th International Symposium 
on Intelligent Systems and Informatics (SISY '07), pp. 237-241, August 2007.

[4] A. Tarko and N. Rouphail, "Travel time data fusion in ADVANCE," in Proceedings of the Pacific Rim TransTech Conference, vol. 1, pp. 36-42, Seattle, Wash, USA, 1993.

[5] R. P. Schäfer, K. U. Thiessenhusen, and P. Wagner, "A traffic information system by means of real-time floating-car data," in Proceedings of the ITS World Congress, 2002.

[6] T. Miwa, T. Sakai, and T. Morikawa, "Route identification and travel time prediction using probe-car data," International Journal of ITS Research, vol. 2, no. 1, pp. 21-28, 2004.

[7] Z. Liyu, W. Huimin, and S. Jianping, "Floating car based realtime-traffic-info collection system in Beijing," Urban Transport of China, vol. 6, no. 1, pp. 77-80, 2008.

[8] Y. Wang, Research on the key technology of large-scale strategic traffic coordination \& control system [Ph.D. thesis], Jilin University, 2009.

[9] J. Zhou and Y. Li, "Hangzhou taxi operating characteristic analysis based on Floating Car Technology," Science \& Technology Information, vol. 3, pp. 120-122, 2011.

[10] C. E. White, D. Bernstein, and A. L. Kornhauser, "Some map matching algorithms for personal navigation assistants," Transportation Research Part C, vol. 8, no. 1-6, pp. 91-108, 2000.

[11] G. Taylor, C. Brunsdon, J. Li, A. Olden, D. Steup, and M. Winter, "GPS accuracy estimation using map matching techniques: applied to vehicle positioning and odometer calibration," Computers, Environment and Urban Systems, vol. 30, no. 6, pp. 757772, 2006.

[12] R. R. Joshi, "A new approach to map matching for in-vehicle navigation systems: the rotational variation metric," in Proceedings of the IEEE Intelligent Transportation Systems Proceedings, pp. 33-38, August 2001.

[13] M. A. Quddus, W. Y. Ochieng, and R. B. Noland, "A general map matching algorithm for transport telematics application," Journal of Navigation, vol. 13, no. 2, pp. 116-119, 2005.

[14] Z. Wang, Z. Li, and L. Wang, "The application of GPS data processing technique in map matching," in Proceedings of the International Conference on Transportation, Mechanical, and Electrical Engineering, pp. 588-591, 2011.

[15] Z. Wang, L. Wang, and J. Wang, "Delay map matching algorithm of mass GPS data based on topological judgment," Journal of Southwest JiaoTong University, vol. 47, no. 5, pp. 861-866, 2012.

[16] H. Takada, Road traffic condition acquisition via mobile phone location referenceing [Ph.D. thesis], University of Waterloo, 2006.

[17] Y. Chen, Traffic flow analysis of urban road network [Ph.D. thesis], ShangHai JiaoTong University, 2008.

[18] B. S. Kerner, C. Demir, R. G. Herrtwich et al., "Traffic state detection with floating car data in road networks," in Proceedings of the 8th International IEEE Conference on Intelligent Transportation Systems, pp. 44-49, September 2005.

[19] Y.-J. Byon, A. Shalaby, and B. Abdulhai, "Travel time collection and traffic monitoring via GPS technologies," in Proceedings of the IEEE Intelligent Transportation Systems Conference (ITSC '06), pp. 677-682, September 2006.

[20] J. Von Neumann and O. Morgenstern, Theory of Games and Economic Behavior (commemorative Edition), Princeton University Press, 2007.

[21] T. C. Koopmans, "Analysis of production as an efficient combination of activities," Activity Analysis of Production and Allocation, vol. 13, pp. 33-37, 1951.
[22] L. A. Zadeh, "Fuzzy sets," Information and Control, vol. 8, no. 3, pp. 338-353, 1965. 


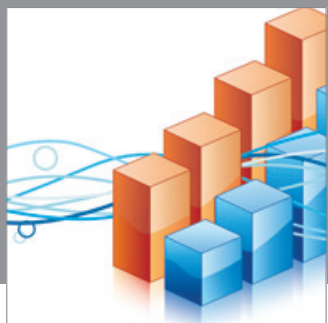

Advances in

Operations Research

mansans

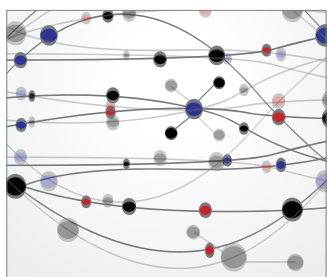

The Scientific World Journal
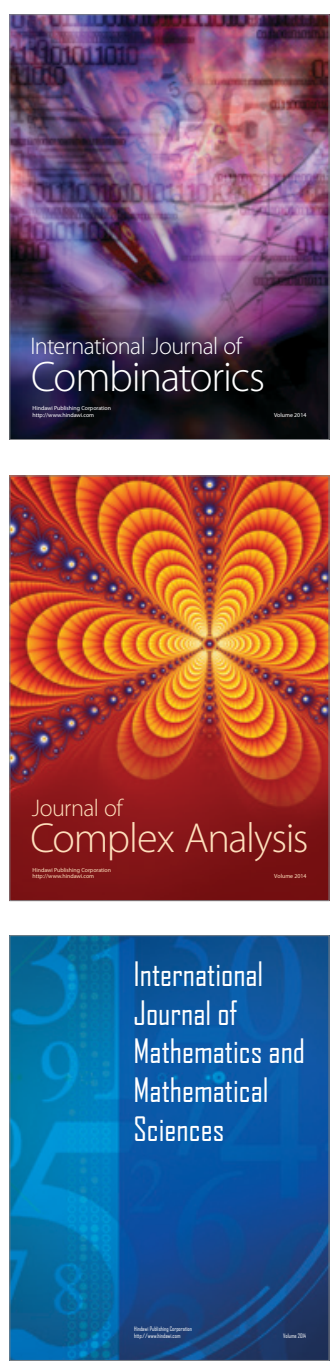
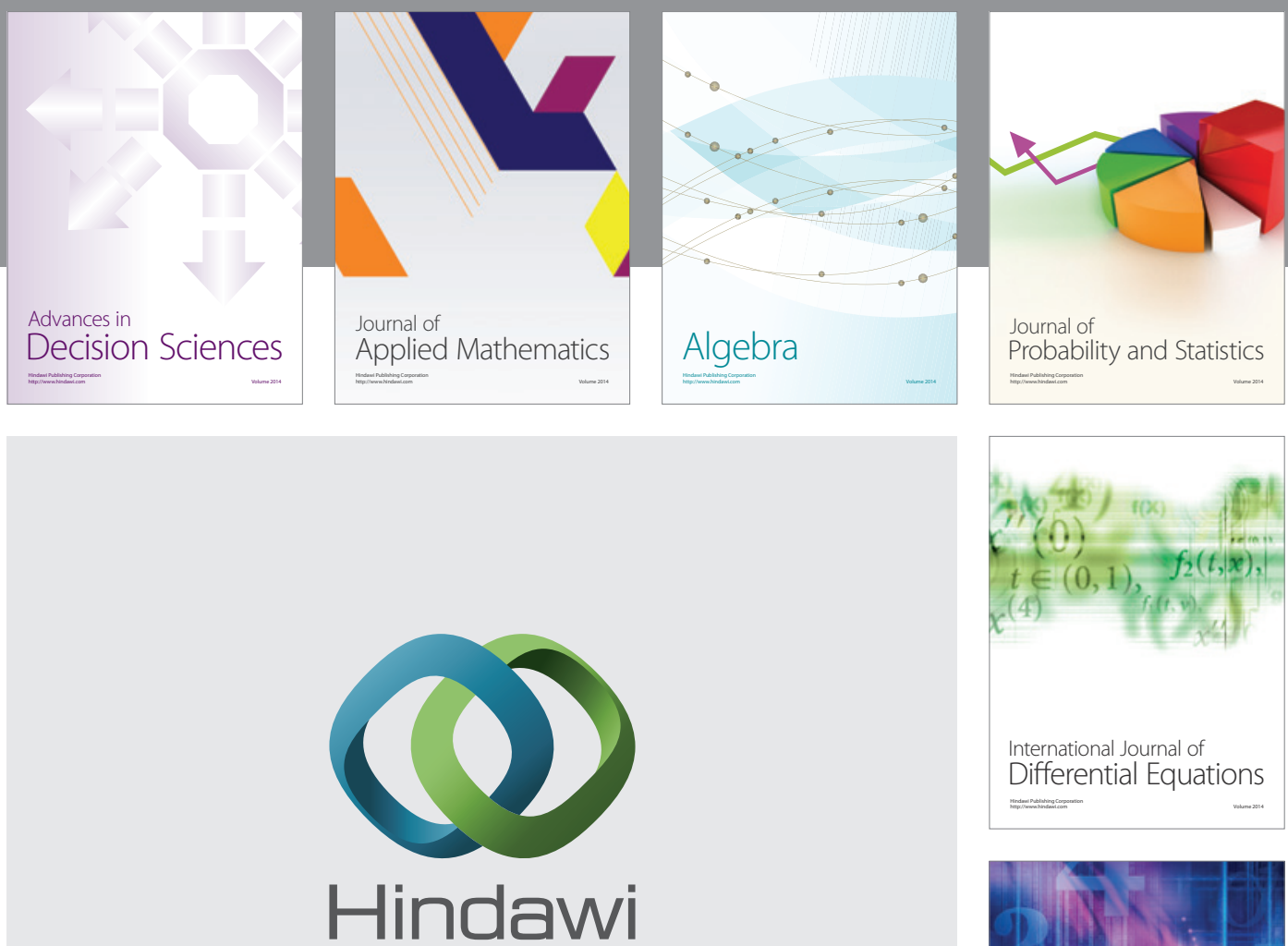

Submit your manuscripts at http://www.hindawi.com
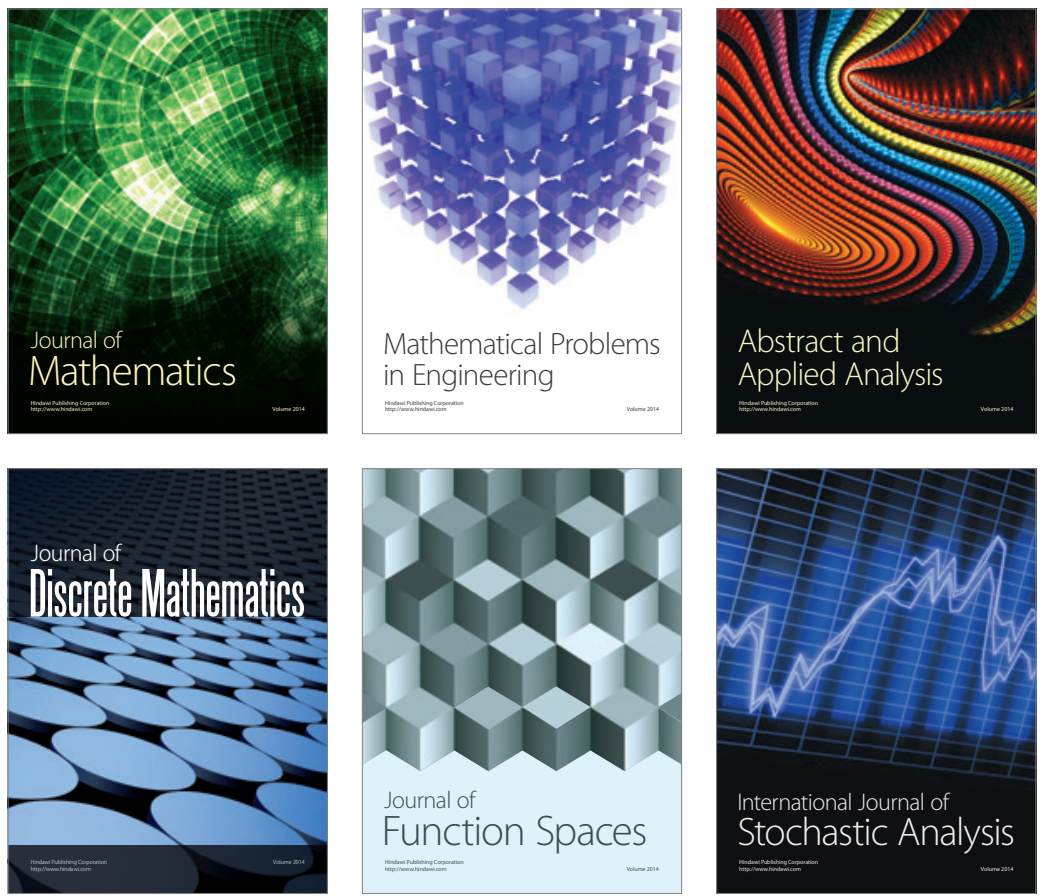

Journal of

Function Spaces

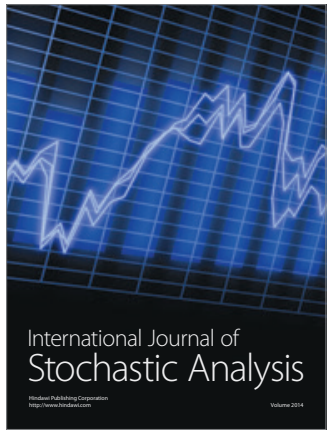

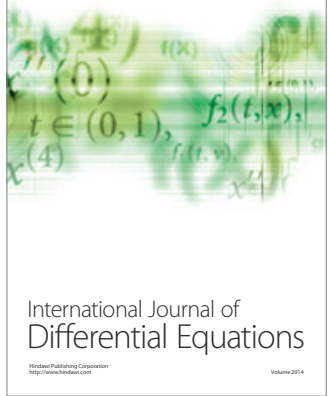
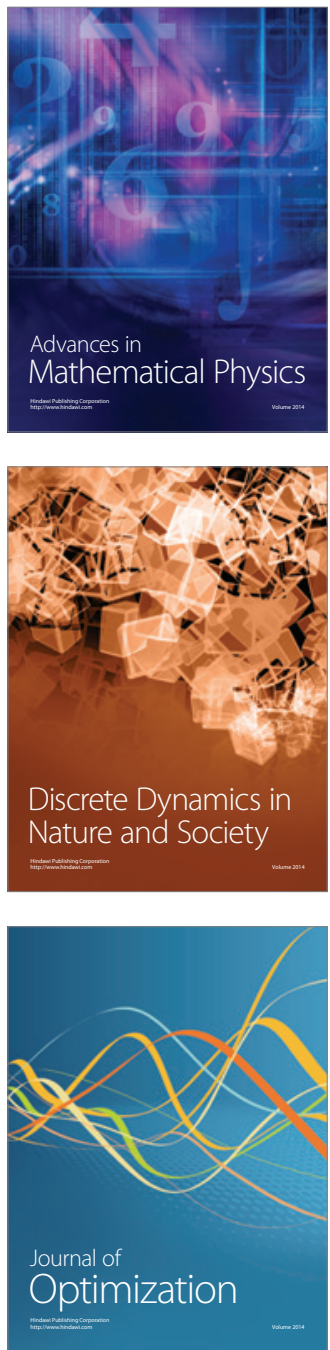